\title{
Analytical Study of Retinal Vessel Changes as a Predictor of Coronary Artery Disease in Elderly Hypertensives Presenting with Angina
}

\author{
Narayanan Balakrishnan' ${ }^{1}$, Arvind Babu², Suhas Prabhakar³, Meera Mohanakumar, Krishnima Raghu ${ }^{5}$, Vishnu S6 \\ ${ }^{1}$ Department of Ophthalmology, Sri Ramachandra Institute of Higher Education and Research, Chennai, \\ Tamilnadu, India. ${ }^{2}$ Department of Ophthalmology, Sri Ramachandra Institute of Higher Education and \\ Research, Chennai, Tamilnadu, India. ${ }^{3}$ Department of Ophthalmology, Sri Ramachandra Institute of Higher \\ Education and Research, Chennai, Tamilnadu, India. ${ }^{4}$ Department of Ophthalmology, Sri Ramachandra \\ Institute of Higher Education and Research, Chennai, Tamilnadu, India. ${ }^{5}$ Department of Ophthalmology, Sri \\ Ramachandra Institute of Higher Education and Research, Chennai, Tamilnadu, India. ${ }^{\text {Department of }}$ \\ Ophthalmology, Sri Ramachandra Institute of Higher Education and Research, Chennai, Tamilnadu, India.
}

\section{ABSTRACT}

\section{BACKGROUND}

Coronary artery disease is an important cause of cardiovascular morbidity and mortality all over the world. Hypertension has been identified as an important risk factor for the development of CAD. Retinal vessel changes can occur in patients with uncontrolled or poorly controlled hypertension and are a predictor of the effect of hypertensive damage in the body. Retina is a place where end artery circulation can be directly visualized and quantifying retinal vessel changes in hypertensive patients presenting with angina can help in determining the risk and identifying those patients who need coronary angiogram especially in a primary care set up as it is an expensive investigation. We wanted to analyse retinal vessel changes in elderly hypertensives presenting with angina and correlate the same with the risk of developing coronary artery disease.

\section{METHODS}

50 elderly hypertensives who presented with acute angina were included in the study and were subjected to a coronary angiogram. They were divided into case and control groups. Those with the disease on angio are taken as cases and those without are considered as controls. The effect of age, sex, BMI, smoking, presence of retinopathy, LVH on ECG, grade of retinopathy were all analysed and correlated with the risk of developing CAD.

\section{RESULTS}

Age, Sex, BMI, smoking, LVH had no relation with development of CAD in our study. Presence of retinopathy had a significant correlation with development of CAD $(\mathrm{p}=$ $0.031, \mathrm{p}<0.05$ ) with OR of 5.81. Commonest grade of retinopathy in our study was Grade 2 and this also showed a significant correlation with the risk of developing CAD.

\section{CONCLUSIONS}

Retinal vessel changes are a significant predictor of risk of developing CAD in elderly hypertensives presenting with angina. The risk of developing CAD was six fold more in those with retinopathy changes and this can help as a screening tool to determine those who need angiography in a primary setting.

\section{KEY WORDS}

Coronary Artery Disease, Hypertensive Retinopathy, Angina
Corresponding Author: Narayanan Balakrishnan, \#1/17, Plot No. 373, $11^{\text {th }}$ Street, Anna Nagar, East Chennai-600102, Tamilnadu, India.

E-mail: narayanbalakrishnan@hotmail.com

DOI: $10.14260 /$ jemds/2020/365

Financial or Other Competing Interests: None.

How to Cite This Article: Balakrishnan N, Babu A, Prabhakar S, et al. Analytical study of retinal vessel changes as a predictor of coronary artery disease in elderly hypertensives presenting with angina. J. Evolution Med. Dent. Sci. 2020;9(21):1663-1668, DOI: 10.14260/jemds/2020/365

Submission 22-03-2020, Peer Review 04-05-2020,

Acceptance 10-05-2020,

Published 25-05-2020.

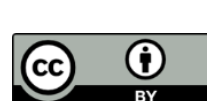




\section{BACKGROUND}

Coronary artery disease (CAD) is the most important cause of cardiovascular mortality all over the world. Coronary artery disease and coronary microvascular dysfunction are important causes of CHD. Cardiovascular disease is currently the leading cause of death in both urban and rural India. Studies by the National $\mathrm{C}$ omission for Macroeconomics and Health, Government of India, suggest that the number of patients with CAD is set to increase to more than 80 million by 2020 , which would represent about $7.6 \%$ of the adult population. The percentage of elderly (N65 years) population of India increased from $6.4 \%$ in 1981 to $7.5 \%$ in 1991.[1]

The prevalence of prehypertension, stage 1 hypertension, and stage 2 hypertension according to Joint National Committee (JNC) VIII classification in patients older than 60 years is estimated to be $30.6 \%, 34.2 \%$, and $16.7 \%$, respectively. Cardiovascular events are also going to be on the rise in the elderly age group with the progressive increasing prevalence of hypertension. Microangiopathy has been observed to play a role in its pathogenesis despite CAD being considered a macrovascular disease. Retina is the only place in the body where microvascular damage can be observed directly.

A fundus (retinal) examination to determine the presence and severity of retinopathy signs has been recommended as a means to determine the presence of end- organ damage in persons with hypertension and to stratify risk. Studies have described that retinal vascular changes predict clinical cardiovascular events, including myocardial infarction in patients with hypertension and diabetes[2][3] We have examined the utility of retinal changes in predicting CAD in an acute setting, when a patient presents to the emergency department with angina. We have tried to assess the utility of hypertensive retinal changes in predicting CAD among elderly patients with hypertension presenting to the emergency department with acute angina. If such a result is established, this will be an immensely useful clinical tool for the primary care physician working in rural India, to prioritize patients requiring immediate intervention.

The retina is the only site in the body where microcirculation can be viewed directly through direct and indirect ophthalmoscopy and recorded through fundus photography. Abnormalities in flow can hence be documented and pathology relations to cardiovascular diseases can be elicited.[4],[5],[6] The different retinal vascular signs, including isolated microaneurysms and haemorrhages, focal, and arterio - venous nicking, are more frequently prevalent in the adult population. They are to be found in retinal photographs in two to 14 percent of the nondiabetic population of adults aged above 40[7],[8],[9] with new signs developing in six to ten percent of people every five years [10],,[11],[12]

\section{Retinal Vascular Pathologies Seen}

- Arteriolar narrowing, which can be focal with Venous.

- Nicking and AV junction changes.

- Superficial flame shaped haemorrhages occurring at RNFL (owing to the anatomy of the nerve fibre layer).

- $\quad$ Micro and macro aneurysms.[13]
- Disc oedema with all of the above Changes.

Histopathological studies have shown that these retinal vessel changes occur from ageing and hypertension.[14],[15] Retinal signs are associated with microvascular pathologies. Retinal arteriole narrowing occurs as arteriolar media thickens and scleroses develops. Sclerotic changes of a like type have been seen in intramyocardial small arterioles, which alongside hypertension show luminal narrowing as in the retina [16][17]. "Increased media to lumen ratio of arteries in subcutaneous fat independently predicts risk of cardiovascular disease events including myocardial infarction. Biopsies of these subcutaneous small arteries (usually obtained from gluteal biopsies) indicate that vascular remodeling is one of the first manifestations of target organ damage, occurring before proteinuria or cardiac hypertrophy, and that it is a reversible, dynamic process."[18],[19]

The amount of remodeling of small arteries is significant over a 10 - year period, worsens with hypertension.[20] Arterioles are similar to small arteries but are less elastic and have fewer muscular fibres. The retinal vessels are a direct window to study these changes.

Retinal vascular signs are associated more commonly with acute lacunar stroke, suggesting focal arteriolar pathologies in the pathogenesis of lacunar stroke, suggesting a potential for retinal imaging to be used in studying small vessel disease.[21]

Retinal vascular changes are an indicator of both the coronary micro- and macro- circulation as the pathologies are paralleled.[22] Retinal arteriolar narrowing is indicative of decreased myocardial perfusion that is seen on cardiac magnetic resonance imaging.[23] "Retinopathy lesions were correlated with coronary artery calcification (measured on cardiac computed tomography scanning) in a dose response manner, with more severe lesions associated with worse coronary artery disease on angiography" Hence a comprehensive anatomical and pathophysiological basis of disease stratification can be done and a direct relation between retinal vasculature and coronary artery disease could be established., A non-invasive retinal assessment may assist CHD risk stratification.

\section{Objectives}

To analyse retinal vessel changes in elderly hypertensives presenting with angina and to establish a correlation between hypertensive retinopathy and coronary heart disease.

\section{METHODS}

This study was conducted in Sri Ramachandra Medical College between 2016-2018 spanning over 2 years. A case control methodology was adopted where the study population contained 50 patients who were elderly patients (age defined $>65$ years) presenting with angina and were divided into 2 groups after a coronary angiogram had been done. One group was diagnosed by the cardiologist who participated in the study to have coronary artery disease and 
the control group was found to be devoid of the disease after being subjected to the angiogram.

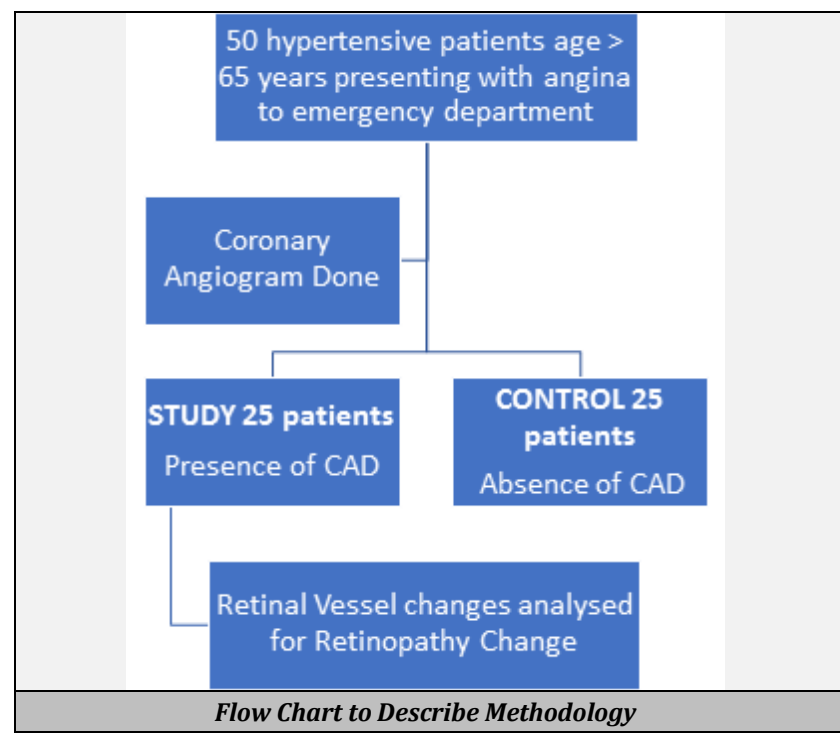

\section{Inclusion Criteria}

1. Age $>65$ years.

2. Those classified to be high or intermediate likelihood group as mentioned

3. Planned for angiography.

4. All patients with prehypertension, stage I hypertension, or stage II hypertension as per JNC VII classification.

An Informed consent was obtained from all patients. The study was approved by the hospital's ethics committee.

\section{Exclusion Criteria}

1. Presence of Diabetes mellitus or prediabetes as per American Diabetes Association.

2. Presence of Metabolic syndrome as elicited by National Cholesterol Education Programme Adult Treatment Panel III guidelines.

3. Presence of overt nephropathy (creatinine N1. $5 \mathrm{mg} /$ dL).

4. Presence of overt proteinuria (albumin- creatinine ratio $\mathrm{N} 300 \mathrm{mg} / \mathrm{g}$ ).

5. Known patients with CAD and were on treatment for the same.

\section{Method of Patient Evaluation}

The study patients were questioned about their age, smoking status, and duration of treatment. Body mass index (BMI) was calculated for all the study patients, and obesity was defined as a BMI greater than $30 \mathrm{Kg} / \mathrm{m}^{2}$.

A 12-lead electrocardiogram was taken and left ventricular hypertrophy was classified according to Romhilt and Estees criteria. The presence or absence of CAD was angiographically determined by the same cardiologist. All study patients underwent angiography. They were then divided into 2 groups one who had evidences of coronary artery disease on the Angiogram and another group who did not have the disease.

1. Retina was examined for all patients in the study by methods described

2. After adequate pupillary dilatation using tropicamide dilating drops, the retina was assessed using direct ophthalmoscope slit lamp biomicroscopy using 78D Volk lenses and indirect ophthalmoscopy using 0D Volk lenses.

3. Fundus photo was taken using a P450 Fundus camera

4. The fundus changes were assessed in the superior temporal field for hypertensive retinal changes.

\section{Ethics Committee Clearance}

This study has been done in accordance with the Helsinki Declaration of 2008 and is approved by the ethics committee of Sri Ramachandra University of higher education and research. There are no financial disclosures to be made for this study.

\section{Statistical Analysis}

All data collected was analysed using chi square X 2 test and student $t$ test. Both the study and control population was analysed and the results were tested for significance to establish a relationship between coronary artery disease and hypertensive retinopathy. The analysis was carried out using SPSS for Windows 10

\section{RESULTS}

The total study population included 50 elderly hypertensive males who presented with angina to the emergency department. The study population was divided into cases and controls on the basis of the presence of coronary artery disease confirmed by angiography.

The study population had 20 males and 5 females and the control population had 16 males and 9 females The average age of the study population was 66.76 years and that of the control population was 64.52 years. The BMI in the study population was 27.26 and that of the control population was 26.26. This did not find a significant correlation. 9 of the 25 $(36 \%)$ in the study population and 13 of the $25(52 \%)$ in the control population were smokers however there was no significant correlation between smoking and the risk of developing CAD. The JNC classification was used to grade the stage of hypertension and Class I was the most common in the study group with $64 \%$ and prehypertension was commonest in the control group with $48 \%$. We found that 21 (84\%) of the study population and $17(68 \%)$ of the control population had significant left ventricular hypertrophy in all patients.

We analysed retinal vessel changes in these patients and classified hypertensive retinopathy changes in all these patients and found that 22 (88\%) of the study population had retinopathy changes and 13 (52\%) of the control population had retinopathy changes and this significantly corelated with the risk of developing coronary artery disease $(\mathrm{p}<0.05)$. [Graph 1]. 


\section{Classification}

\section{- Keith-Wagener-Barker classification}

\begin{tabular}{|c|c|}
\hline Grade & Description \\
\hline Grade 1 & $\begin{array}{l}\text { Slight narrowing, sclerosis, and tortuosity of the retinal arterioles; mild, } \\
\text { asymptomatic hypertension }\end{array}$ \\
\hline Grade 2 & $\begin{array}{c}\text { Definite narrowing, focal constriction, sclerosis, and AV nicking; blood } \\
\text { pressure is higher and sustained; few, if any, symptoms referable to blood } \\
\text { pressure }\end{array}$ \\
\hline Grade 3 & $\begin{array}{l}\text { Retinopathy (cotton-wool patches, arteriolosclerosis, hemorrhages); blood } \\
\text { pressure is higher and more sustained; headaches, vertigo, and } \\
\text { nervousness; mild impairment of cardiac, cerebral, and renal function }\end{array}$ \\
\hline Grade 4 & $\begin{array}{c}\text { Neuroretinal edema, including papilledema; Siegrist streaks, Elschnig } \\
\text { spots; blood pressure persistently elevated; headaches, asthenia, loss of } \\
\text { weight, dyspnea, and visual disturbances; impairment of cardiac, cerebral, } \\
\text { and renal function }\end{array}$ \\
\hline
\end{tabular}

Table 1. The Retinopathy Changes were Interpreted Using the Keith-Wagner Barker Classification of Hypertensive Retinopathy
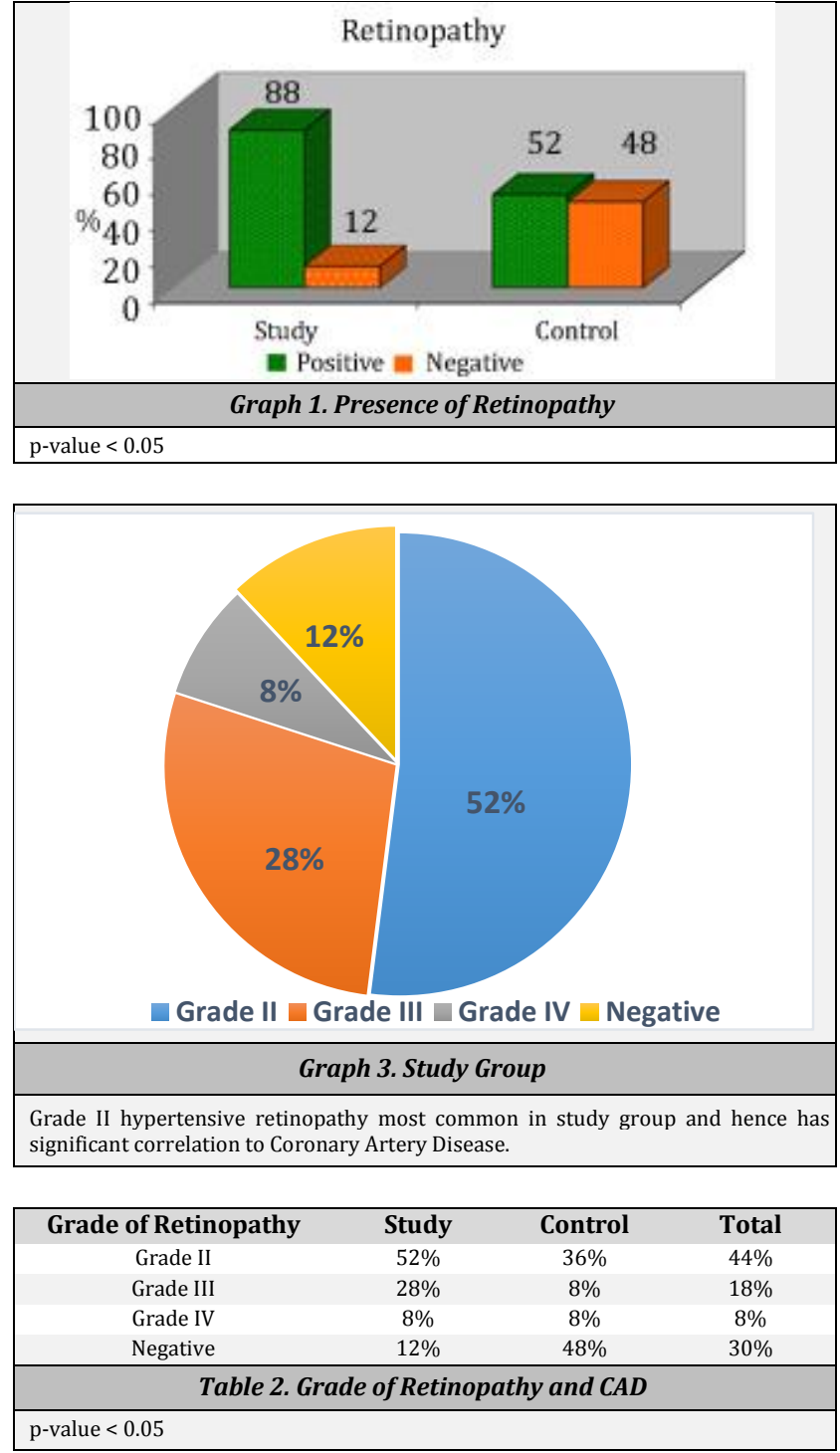

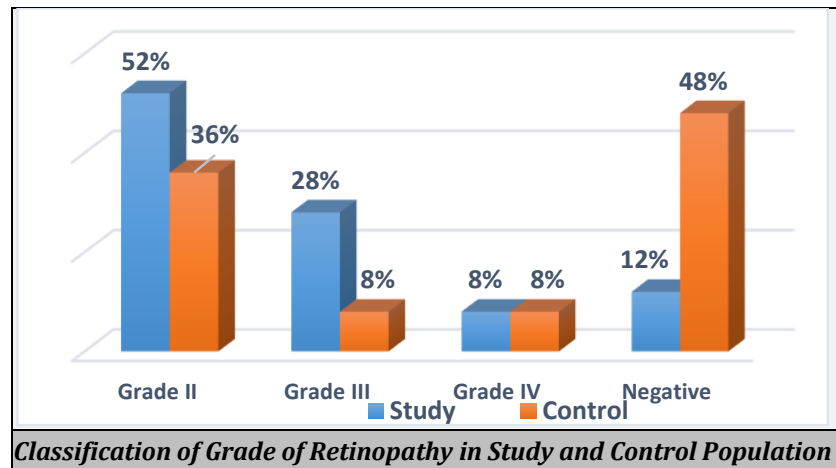

We also calculated the odds ratio for developing CAD in the presence of Hypertensive retinopathy changes and found an OR 5.81 in the study group.

We graded the severity of retinopathy as mentioned above and found that Grade II was the commonest grade found in both the study and control population. [Table 2] [Graph 2][Graph 3].

\section{DISCUSSION}

This study had a population that was more than 65 years of age in both the study and control group. The average age of the study population was 67.75 years. The prevalence of CAD in south India was found to be $68+/-3$ years which was proven by Shanta et al in 2012 .

There was no specific sex predilection seen in this study both males and females were equally affected. Some studies have shown an interesting observation that retinal arteriolar narrowing was related to risk of CAD more in women than in men. Coronary artery disease is known to be strongly associated with smoking however in our study this feature 
was not established as it was a case control study containing only 25 members in study and control group.[24]

It was also observed that hypertensives who fell in the Class II of JNC for Hypertension with a long standing duration of hypertension were most likely to develop coronary artery disease.[25] Most of them were under treatment for hypertension but were either poor with their medical compliance or had discontinued treatment over the years. This result is consistent with a number of other studies.

The comparison of Left ventricular hypertrophy in the 2 groups also showed an association with coronary artery disease however this was not a significant association because both the study and control groups were known hypertensives and it is established that chronic hypertension is associated with left ventricular hypertrophy.[26]

In our study we divided elderly hypertensive patients who presented with angina into 2 groups one with coronary artery disease after an angiogram was done and the other group that acted as the control did not have the disease.

\section{Analysis of Retinal Vessel Changes}

In the Study Group

- $52 \%$ of the population had grade II hypertensive retinopathy.

- $28 \%$ of the population had grade III hypertensive retinopathy.

- $8 \%$ of the population had grade IV hypertensive retinopathy.

- $12 \%$ of the population did not have retinopathy.

In the Control Group

- $36 \%$ had grade II hypertensive retinopathy.

- $8 \%$ had grade III hypertensive retinopathy.

- $8 \%$ had grade IV hypertensive retinopathy.

- $48 \%$ of the population did not have any signs of hypertensive retinopathy.

So roughly $88 \%$ of the study population had retinopathy changes and this on Chi square testing this had a significance of $\mathrm{p} 0.031(\mathrm{p}<0.05)$ establishing a strong association between hypertensive retinopathy and coronary artery disease. This proves that hypertensive retinopathy could be an early predictor of coronary artery disease in elderly hypertensives presenting with angina.

A prevalence based study done by Shanta Et al in 2012 they compared the retinal vessel changes in hypertensives presenting with angina and found that the LR + value was 3.92 and LR - was 0.52 which was a fairly significant number showing that retinopathy is a fair predictor of coronary artery disease in elderly hypertensives.[27] In our study, which is a case control study, a strong association has been established.

Wang et al analysed the retinal vessel calibre in hypertensives and used it to predict the morbidity of coronary artery disease and found that a decreased vessel calibre in arteries and an increase in artery to vein ratio was directly associated with coronary artery disease.[28] This was also seen grossly in our study which used the Keith Wagner and Barker's classification which was based on arteriolar narrowing secondary to hypertension and the crossing changes that occurred with it.[29]

Wong TY, et al showed the association between retinal changes and coronary artery calcification, which was used as a marker of subclinical CAD. Also, they showed that this finding was consistent in patients with or without diabetes or hypertension.[30]

We also found that there was a 5.81 odds ratio in the study population which means that there is a 6 fold increased chance of developing coronary artery disease if there is presence of hypertensive retinopathy in elderly hypertensives.

We also observed that $52 \%$ of the population had grade II hypertensive retinopathy, which dominated the presentation of retinopathy in the observed cases proving that Grade II hypertensive retinopathy with $\mathrm{LVH}$ has a significant correlation with coronary artery disease with elderly hypertensives presenting with angina. This result was comparable to the study conducted by Gee-Hee Kim, et al.

In this study they found that the grade of hypertensive retinopathy was related to age, duration of hypertension, coronary artery disease (CAD), and left ventricular hypertrophy (LVH). The prevalence of LVH and CAD in Grade II was significantly higher than in Grade I and Grade 0. Thus, there was a strong correlation between grade II hypertensive retinopathy and Coronary artery disease much like in our study.

\section{Limitations}

Sample Size is only 50 with 25 cases and 25 controls. This limited sample size deterred us from establishing other risk factors that have been found to be significant in other studies, example smoking, BMI. However, the sample size was calculated for this case control methodology using statistical tools and is adequate to establish a numerically significant result.

We were not able to calibrate the vessel dimensions due to certain technical and financial constraints and the determinants of vessel changes are based on broad observations. Vessel Caliber and amount of narrowing would enable us to predict the changes more accurately as done by certain other studies. However, the classification used to determine retinal vasculature changes are standard and were done by one observer, hence eliminating intra observer bias.

\section{CONCLUSIONS}

The retinal vessel changes that were observed with hypertensive retinopathy in the study population established a strong association with coronary artery disease. There was a 6-fold increase in risk of developing coronary artery disease in patients with hypertensive retinopathy. Grade II hypertensive retinopathy had the highest association with developing Coronary Artery Disease and is a significant and red flag sign in elderly hypertensive patients with angina. Retinal vascular changes secondary to hypertension are an important predictor of coronary artery disease in elderly hypertensives presenting with angina and can be used as a screening tool especially in primary care centres where a 
decision to do an angiogram is cost dependant and this is a significant conclusion of this study.

\section{REFERENCES}

[1] Indrayan A. Forecasting cardiovascular disease cases and associated mortality in India. New Delhi: National Commission for Macroeconomics and Health, Government of India, 2004.

[2] Wong TY, Mitchell P. Hypertensive retinopathy. N Engl J Med 2004;351(22):2310-7.

[3] Wong TY, Klein R, Sharrett AR, et al. Retinal arteriolar narrowing and risk of coronary heart disease in men and women. The Atherosclerosis Risk in Communities Study. JAMA 2002;287(9):1153-9.

[4] Wong TY, McIntosh R. Systemic associations of retinal microvascular signs: a review of recent population based studies. Ophthalmic Physiol Opt 2005;25(3):195204.

[5] Wong TY, McIntosh R. Hypertensive retinopathy signs as risk indicators of cardiovascular morbidity and mortality. Br Med Bull 2005;73-74:57-70.

[6] Tso MO, Jampol LM. Pathophysiology of hypertensive retinopathy. Ophthalmology 1982;89(10):1132-45.

[7] Wong TY. Is retinal photography useful in the measurement of stroke risk? Lancet Neurol 2004;3(3):179-83.

[8] Mosseri M, Yarom R, Gotsman MS, et al. Histologic evidence for small-vessel coronary artery disease in patients with angina pectoris and patent large coronary arteries. Circulation 1986;74(5):964-72.

[9] Klein R. Retinopathy in a population-based study. Trans Am Ophthalmol Soc 1992;90:561-94.

[10] Klein R, Klein BE, Moss SE, et al. Blood pressure, hypertension and retinopathy in a population. Trans Am Ophthalmol Soc 1993;91:207-26.

[11] Klein R, Klein BE, Moss SE. The relation of systemic hypertension to changes in the retinal vasculature: the Beaver Dam Eye Study. Trans Am Ophthalmol Soc 1997;95:329-50.

[12] Klein R, Myers CE, Lee KE, et al. 15 - year cumulative incidence and associated risk factors for retinopathy in non-diabetic persons. Arch Ophthalmol 2010;128(12):1568-75.

[13] Cugati S, Cikamatana L, Wang JJ, et al. Five- year incidence and progression of vascular retinopathy in persons without diabetes: the Blue Mountains Eye Study. Eye (Lond) 2005;20(11):1239-45.

[14] Rizzoni D, Porteri E, Boari GEM, et al. Prognostic significance of small - artery structure in hypertension. Circulation 2003;108(18):2230-5.

[15] Kralev S, Zimmerer E, Buchholz P, et al. Microvascular retinal changes in patients presenting with acute coronary syndromes. Microvascular Research 2010;79(2):150-3.
[16] Schiffrin EL, Park JB, Intengan HD, et al. Correction of arterial structure and endothelial dysfunction in human essential hypertension by the angiotensin receptor an tagonist losartan. Circulation 2000;101(14):1653-9.

[17] Park JB, Schiffrin EL. Small artery remodeling is the most prevalent (earliest?) form of target organ damage in mild essential hypertension. J Hypertens 2001;19(5):921-30.

[18] Lindley RI, Wang JJ, Wong MC, et al. Retinal microvasculature in acute lacunar stroke: a crosssectional study. Lancet Neurol 2009;8(7):628-34.

[19] Doubal FN, Macgillivray TJ, Hokke PE, et al. Differences in retinal vessels support a distinct vasculopathy causing lacunar stroke. Neurology 2009;72(20):1773-8.

[20] Wang L, Wong TY, Sharrett AR, et al. Relationship between retinal arteriolar narrowing and myocardial perfusion: multi - ethnic study of atherosclerosis. Hypertension 2008;51(1):119-26.

[21] Wong TY, Cheung N, Islam FMA, et al. Relation of retinopathy to coronary artery calcification: the multiethnic study of atherosclerosis. Am J Epidemiol 2008;167(1):51-8.

[22] Tedeschi-Reiner E, Strozzi M, Skoric B, et al. Relation of atherosclerotic changes in retinal arteries to the extent of coronary artery disease. Am J Cardiol 2005;96(8):1107-09.

[23] Kim GH, Youn HJ, Kang S, et al. Relation between Grade II hypertensive retinopathy and coronary artery disease in treated essential hypertensives. Clinical and Experimental Hypertension 2010;32(7):469-73.

[24] Shah PK, Helfant RH. Smoking and coronary artery disease. Chest 1988;94(3):449-52.

[25] Chobanian AV, Bakris GL, Black HR, et al. Seventh report of the Joint National Committee on Prevention, Detection, Evaluation and Treatment of High Blood Pressure. Hypertension 2003;42(6):1206-52.

[26] Pepine CJ, Kowey PR, Kupfer S, et al. Predictors of adverse outcome among patients with hypertension and coronary artery disease. Journal of the American College of Cardiology 2006;47(3):547-51.

[27] Shantha GPS, Srinivasan Y, Kumar AA, et al. Can retinal changes predict coronary artery disease in elderly hypertensive patients presenting with angina? The American Journal of Emergency Medicine 2010;28(5):617-21.

[28] Wang JJ, Liew G, Wong TY, et al. Retinal vascular calibre and the risk of coronary heart disease - related death. Heart 2006;92(11):1583-7.

[29] Downie LE, Hodgson LAB, Dsylva C, et al. Hypertensive retinopathy: comparing the Keith-Wagener-Barker to a simplified classification. J Hypertens 2013;31(5):960-5.

[30] Wong TY, Klein R, Sharrett AR, et al. Retinal arteriolar narrowing and risk of coronary heart disease in men and women. The Atherosclerosis Risk in Communities Study. JAMA 2002;287(9):1153-9. 